\title{
Do junior doctors at the Manchester Royal Infirmary know how to refer inpatients to medical specialties?
}

\author{
Authors: Sabine Jamal, Catriona Boyd, Basim Ali and Elinor Shuttleworth
}

\section{Introduction}

Due to the structure of training posts, junior doctors frequently rotate through different trusts. Although most trusts conduct induction periods to mitigate this, many junior doctors struggle to perform everyday tasks such as referring their patients to other medical specialties. This was particularly problematic at the Manchester Royal Infirmary (MRI) where no formal information was provided outlining the referral system. The problem was exacerbated by the fact that each inpatient specialty has a unique method of referrals. All these systems, combined with a lack of information provided, meant that doctors were wasting significant amounts of time every day trying to find out how to refer their patients. The aim of this project was to highlight the problem faced by junior doctors when trying to find out how to refer their patients and come up with a simple, yet effective, solution.

\section{Materials and methods}

Phase 1: Junior doctors from FY1 to pre-registrar levels were surveyed and asked whether they knew how to refer to inpatient medical specialties at MRI. We asked them to specify whether this information was sourced formally through trust systems or informally via their colleagues.

Intervention: A formal document outlining how every medical specialty at MRI was produced and disseminated to all doctors within the trust via email, intranet and physical copies on both medical and surgical wards.

Phase 2: Junior doctors of the same grades were then resurveyed and asked whether or not they knew how to refer to inpatient specialties. Again, they were asked how this information was gathered. We also assessed any time saving benefits to the document.

\section{Results and discussion}

During Phase 1 it was found that, not only did $91 \%$ of junior doctors surveyed agree that figuring out how to refer their patients to other specialties wasted a significant amount of time, but $98 \%$ agreed that a formal document outlining the referral pathways would save them time during the working day.
Phase 2 (post-intervention) results showed that $96 \%$ of junior doctors surveyed were aware of the intervention (formal document), with the same amount agreeing that it saves them a significant amount of time during their day. Additionally, all junior doctors audited said that they would use the document in the future.

\section{Conclusion}

As we can see from the results, it was clear that, prior to any intervention, junior doctors from a range of grades and specialties were either not aware of how to refer or had gathered this information informally. This confirmed our hypothesis that there was an issue in this area.

Following the intervention, Phase 2 results showed that the majority of junior doctors now knew how to refer their patients. Additionally, they were aware that a formal document outlining the process existed and agreed that it saved them significant amounts of time.

In conclusion, referrals are an important part of a junior doctor's daily role. This project not only highlighted a major issue in performing this task but successfully implemented an intervention, which alleviated the problem in the Manchester Royal Infirmary. 